\title{
Escória de aciaria na redução da solubilidade e lixiviação de manganês em áreas contaminadas
}

\author{
Imaking slag to reducing the solubility and leaching of manganese in contaminated areas
}

\author{
Ana Carolina Callegario Pereira ${ }^{\mathrm{I}}$ Nelson Moura Brasil do Amaral Sobrinho ${ }^{\mathrm{II}}$ \\ Nelson Mazur ${ }^{\mathrm{II}}$ Ricardo Valcarcel ${ }^{\mathrm{III}}$ Fabiana Soares dos Santos ${ }^{\mathrm{IV}}$
}

RESUMO

A escória de aciaria é um rejeito da produção do aço e possui propriedade corretiva da acidez do solo, podendo reduzir consequentemente a mobilidade de elementos traço. O trabalho objetivou avaliar a capacidade do resíduo industrial escória de aciaria em reduzir a solubilidade e lixiviação de $\mathrm{Mn}$ em resíduos contaminados. No Laboratório de Poluição de Solos da UFRRJ (Seropédica-RJ), foram realizados ensaios de lixiviação em colunas usando três resíduos contaminados com Mn, denominados G1, G2 e G3 e duas doses de resíduo inertizante (escória de aciaria). Nas colunas, foi aplicado um volume de água conhecido e constante que simula a precipitação máxima ocorrida nos últimos 17 anos na região onde foi coletado o resíduo. A maior dose do resíduo de escória de aciaria (6\%) reduziu a solubilidade do $\mathrm{Mn}$ a valores inferiores ao limite máximo permitido pela $A B N T$, nos grupos G1, G2 e G3, sendo que o tratamento com 6\% escória de aciaria + barreiras químicas foi o mais eficiente na redução da lixiviação de $\mathrm{Mn}$ em todos os grupos de resíduos estudados. Portanto, os resultados evidenciaram a eficiência da escória de aciaria na redução da solubilidade e lixiviação do $\mathrm{Mn}$, em todos os resíduos.

Palavras-chave: resíduos industriais, contenção química, elemento traço, material inertizante.

\section{ABSTRACT}

Steelmaking slag is a waste of steel production and has owned corrective soil acidity and may consequently reduce the mobility of trace elements. The research aimed the capacity of industrial waste steelmaking slag to reduce the solubility and lixiviation of $\mathrm{Mn}$ in contaminated soil. In the Soil Pollution
Laboratory of the UFRRJ (Seropédica-RJ), leaching experiments were carried using three different residues contaminated with $\mathrm{Mn}$, and two doses of the inertizant residues steelmaking slag. In the columns were applied a known and constant volume of water, established to simulate the maximum precipitation occurred in the last 17 years, in the region where the residue was collected. The highest dose of steelmaking slag (6\%) reduced the Mn solubility below the maximum allowed by ABNT, for G1, G2 and Ge, and the treatment with $6 \%$ steelmaking slag + barriers chemical was the most effective in reducing the leaching of $\mathrm{Mn}$ in all groups. Therefore, the results showed the efficiency of steelmaking slag in the reduction of Mn solubility and lixiviation in all residues.

Key words: industrial wastes, chemical containment, trace elements, inertizant material.

\section{INTRODUÇÃO}

O elevado volume de resíduos gerados na siderurgia requer a permanente busca de alternativas para o seu destino, que resultem em uma menor agressão ambiental e um menor dispêndio de recursos. $\mathrm{O}$ interesse na remediação de solos e substratos contaminados por elementos traço é relevante em função dos seus efeitos negativos. Elementos traço no solo podem comprometer a cadeia alimentar, assim como serem lixiviados ao lençol freático, ampliando a área de contaminação e prejudicando a qualidade das águas subterrâneas (FUNGARO et al., 2004).

O Mn é um elemento essencial para os microrganismos do solo e plantas. No entanto,

IDepartamento de Formação Profissional do Centro de Tecnologias e Engenharias, Centro Universitário de Volta Redonda (UniFOA), Av. Paulo Erlei Abrantes, 1325, 27240-560, Volta Redonda, RJ, Brasil. E-mail: acallegario@yahoo.com.br. Autor para correspondência. IIDepartamento de Solos, Universidade Federal Rural do Rio de Janeiro (UFRRJ), Seropédica, RJ, Brasil.

IIIDepartamento de Ciências Ambientais, UFRRJ, Seropédica, RJ, Brasil.

${ }^{\text {IV }}$ Departamento de Engenharia de Agronegócios, Universidade Federal Fluminense (UFF), Volta Redonda, RJ, Brasil. Recebido 23.08.12 Aprovado 29.08.13 Devolvido pelo autor 02.12.13 CR-2012-0733.R3 
concentrações acima dos limites ideais podem trazer sintomas de toxicidade aos seres vivos, sendo a redução do $\mathrm{pH}$ um dos fatores que mais contribuem para o aumento da sua biodisponibilidade (ALLOWAY, 1995).

A imobilização química é uma forma de contenção de Mn e consiste em minimizar a migração de contaminantes e sua toxicidade (BASTA \& MCGOWEN, 2004). Os produtos utilizados para essa imobilização podem ser considerados agentes inertizantes. $\mathrm{O}$ uso de agentes inertizantes em solos contaminados geralmente tem o intuito de aumentar a complexação de elementos traço na superfície das partículas do solo, principalmente naquelas compostas de óxidos, hidróxidos e óxi-hidróxidos de Fe e Al, e de precipitá-los na solução (CASTALDI et al., 2005; TREVIZAM et al., 2010). Segundo a estratégia de contenção química, não ocorre uma redução efetiva do contaminante, mas os riscos à saúde e ao ambiente são minimizados a níveis aceitáveis por uma manipulação física ou química da área contaminada (MESQUITA et al., 2006).

Segundo AGOURAKIS et al. (2006), a solubilidade dos metais tóxicos é estabelecida principalmente pelas propriedades físico-químicas do solo, sendo os parâmetros de maior influência o pH, a presença de substâncias orgânicas e inorgânicas formadoras de complexos e, também, a concentração e espécie química do metal presente. Entre as propriedades do solo, o pH é o parâmetro que mais influencia a solubilidade de metais, sendo que o $\mathrm{pH}$ neutro e ligeiramente alcalino favorece a imobilização de elementos traço catiônicos no solo, pois o metal está sujeito à precipitação com hidróxidos, carbonatos e fosfatos, diminuindo sua biodisponibilidade e lixiviação para as águas subterrâneas (AGOURAKIS et al., 2006).

A escória de aciaria é um rejeito da produção do aço e possui, em sua composição, elevados teores de óxido e silicato de cálcio, além de propriedade corretiva de acidez do solo (PRADO et al., 2003). Com o objetivo de estudar o efeito da escoria de aciaria na produção vegetal, MAGALHÃES et al. (2012) concluíram que o uso desse rejeito, por causa do seu elevado pH, foi eficiente na redução da biodisponibilidade de elementos traço, propiciando um melhor desenvolvimento de plantas de eucalipto.

O trabalho objetivou avaliar a capacidade do resíduo industrial escória de aciaria, oriundo da CSN, em reduzir a solubilidade e lixiviação do Mn em resíduos contaminados.

\section{MATERIAL E MÉTODOS}

Os resíduos contaminados utilizados neste trabalho são provenientes de escavações de solo, realizadas para instalar colunas de brita com o objetivo de aumentar a sustentação do pátio de minério do Porto de Itaguaí (Itaguaí-RJ). A área está localizada próxima ao aterro de resíduos perigosos, com elevadas concentrações de Mn e outros elementos traço (SANTOS et al., 2007) gerados pela Cia Mercantil e Industrial Ingá. Esses resíduos foram dispostos em 9 pilhas, no pátio da CSN, totalizando um volume de $149.650 \mathrm{~m}^{3}$, o que inviabilizava a continuação das obras de ampliação da capacidade de suporte do Porto.

Foram coletadas amostras das nove pilhas de resíduos, repartindo-se as pilhas em três partes iguais, extraindo-se amostras em três alturas relativas aos terços inferior, médio e superior das pilhas, segundo Norma 10007 (ABNT, 2004a). Essas nove pilhas de resíduos foram agrupadas por semelhanças nas características físicas e químicas (análise granulométrica, $\mathrm{pH}$ e teores de Mn no solubilizado), resultando em três grupos de resíduos (G1, G2 e G3) nos quais foram realizados os estudos.

Após a homogeneização dos setores para a formação dos grupos (G1, G2 e G3), esses resíduos foram umedecidos a $80 \%$ da capacidade de campo e permaneceram incubados por 10 dias. Amostras foram coletadas, secas ao ar, destorroadas, trituradas em almofariz de ágata e peneiradas, utilizando uma peneira com abertura de $2 \mathrm{~mm}$ para determinação da análise granulométrica, segundo (Tabela 1). Para determinação de teores pseudototais de elementos traço, essas amostras foram passadas em peneira com abertura de $210 \mu \mathrm{m}$ para a realização da digestão com água régia (EPA, 1986). Foi também realizado ensaio de solubilização, segundo a norma NBR 10006 (ABNT, 2004b), na qual se verificou que as concentrações de $\mathrm{Mn}$ no extrato solubilizado encontravam-se acima dos limites permitidos pela ABNT, 2004c (Tabela 1). O menor valor de $\mathrm{pH}$ observado no G1 $(3,9)$ favoreceu maior solubilização de Mn presentes em sua constituição em relação aos resíduos dos grupos G2 e G3 (Tabela 1).

\section{Caracterização do resíduo inertizante}

Como inertizante, foi usado o resíduo escória de aciaria (EA), que é um resíduo alcalino, com $\mathrm{pH}$ de 12,3, contendo: $42 \%$ de $\mathrm{CaO}$; 4,7\% MgO; $36 \% \mathrm{SiO}_{2}$, e tem a função de reduzir a solubilidade de elementos traço catiônicos pelo aumento do $\mathrm{pH}$. Amostras desse resíduo foram trituradas em 
Tabela 1 - Teores pseudototais de elementos traço $\left(\mathrm{mg} \mathrm{kg}^{-1}\right)$, pH, concentração de Mn no extrato solubilizado (NBR 10006) e composição granulométrica nos resíduos removidos do pátio de estocagem de minério da CSN (G1, G2 e G3) ${ }^{1}$.

\begin{tabular}{|c|c|c|c|c|c|c|c|c|c|}
\hline Grupos & $\mathrm{Zn}$ & Mn & $\mathrm{Pb}$ & $\mathrm{Cd}$ & Mn ${ }^{\text {solubilizado }}$ & $\mathrm{pH}$ em $\mathrm{H}_{2} \mathrm{O}$ & areia & silte & Argila \\
\hline G1 & 814,0 & 302,5 & 54,0 & 1,8 & 21,1 & 3,9 & 54 & 25 & 21 \\
\hline G2 & 1287,0 & 265,0 & 31,6 & 0,5 & 18,5 & 4,6 & 55 & 20 & 25 \\
\hline G3 & 739,0 & 410,0 & 34,6 & 3,3 & 10,0 & 6,7 & 62 & 21 & 17 \\
\hline
\end{tabular}

${ }^{1}$ Os valores correspondem à média de cinco repetições.

Limite de Mn no solubilizado (0,1 $\left.\mathrm{mg} \mathrm{kg}^{-1}\right)$ segundo ABNT, 2004c.

almofariz de ágata e passadas por peneira de malha de nylon com abertura de $210 \mu \mathrm{m}$ para a digestão com água régia (EPA, 1986). Os teores pseudototais de $\mathrm{Zn}$, $\mathrm{Pb}$ e Mn na escória de aciaria foram: 109,50; 77,92 e 195,00 $\mathrm{mg} \mathrm{kg}^{-1}$, respectivamente.

Tratamentos para inertização de Mn

Para se definir a proporção adequada da EA, foi realizada uma curva de neutralização, que consistiu em adicionar quantidades crescentes de EA ao solo contaminado, na proporção de: $1 \%$; 2\%; 3\%; 4\%; 5\%, e 6\% do resíduo alcalino. Essa mistura permaneceu incubada com uma umidade correspondente a 70\% da capacidade de campo por 7 dias até a estabilização do $\mathrm{pH}$. Verificou-se que, para atingir os valores de pH 6 e 7, foi necessário adicionar as doses de $4 \%$ e $6 \%$ de EA, respectivamente, para os substratos G1 e G2.

Os tratamentos utilizados foram: 100\% Grupo (testemunha); 96\% Grupo + 4\% EA; 94\% Grupo + 6\% EA; e $94 \%$ Grupo + 6\% EA + Barreiras químicas de EA (6\%). Para cada tratamento, foram realizadas cinco repetições.

As barreiras químicas correspondem a camadas de inertizantes adicionadas entre camadas de tratamentos nas colunas. Sua função foi de potencializar o efeito dos tratamentos na redução da solubilidade de $\mathrm{Mn}$, quando este for lixiviado pela passagem da água no sistema.

Foram retiradas amostras de todos os tratamentos, com exceção dos que contêm as barreiras químicas, para realização do ensaio de solubilização, segundo NBR 10006 (ABNT, 2004b).

\section{Ensaio de lixiviação em coluna}

O ensaio de lixiviação de Mn foi realizado em colunas de vidro, com a base em forma de "V" para facilitar o escoamento da água adicionada. Cada coluna possui seção transversal quadrada de lado $0,15 \mathrm{~m}$ e altura $0,7 \mathrm{~m}$. Uma mangueira plástica foi colocada no fundo da coluna, para a saída de percolado. Nas colunas previamente lavadas e descontaminadas, foram adicionadas brita para evitar perda de substrato pela base. A brita também foi lavada e descontaminada com solução de ácido clorídrico 5\%.

Cada coluna foi preenchida com uma massa de $6 \mathrm{~kg}$ de resíduo tratado. Nos tratamentos em que foram utilizadas barreiras químicas, estas foram dispostas da seguinte maneira: após a colocação de $2 \mathrm{~kg}$ de resíduo tratado, foi adicionada $50 \%$ da dose de EA, isto é, 3\%, uniformemente sobre esta camada. Posteriormente, mais $2 \mathrm{~kg}$ de resíduo tratado e os $3 \%$ restantes da dose de EA e, finalmente, sobre esta barreira, a última camada de $2 \mathrm{~kg}$ de resíduo tratado. Cada porção de um quilo foi homogeneizada em um saco plástico, por meio de movimentos circulares, e transferida para dentro da coluna.

Ao se atingir $6 \mathrm{~kg}$ em cada coluna, foi adicionada água referente a $80 \%$ da capacidade de campo e o material permaneceu incubado por dez dias. Para a determinação da quantidade de água a ser percolada nas colunas, foi realizado um estudo de distribuição da precipitação dos últimos 17 anos na área para caracterizar situações de precipitação pluviométrica mais críticas, que poderiam influenciar a lixiviação de elementos químicos no solo. Foi analisado um período de retorno que variou de 2 a 50 anos e estabeleceu-se 50 anos como um período mais crítico. De acordo com os dados climatológicos, foi adicionado, no primeiro dia do ensaio, uma quantidade de água equivalente a uma chuva de $250 \mathrm{~mm} \mathrm{dia}^{-1}$ (5,6L coluna $\left.{ }^{-1} \mathrm{dia}^{-1}\right)$, correspondente ao volume de precipitação máxima, e 1/5 deste volume a cada dia $\left(1,1 \mathrm{~L}\right.$ coluna ${ }^{-1}$ dia $\left.^{-1}\right)$, durante cinco dias, totalizando seis dias de ensaio para os três grupos (G1, G2 e G3).

Para a adição da água no sistema foi determinada uma altura constante, chamada de “cabeça hidráulica”, para manter a mesma pressão da água em cada coluna. A cabeça hidráulica ficou a $5 \mathrm{~cm}$ da camada superficial dos tratamentos nas colunas, e o 
volume de água a ser utilizado a cada dia foi colocado de maneira constante, mantendo a cabeça hidráulica até a adição total da água.

Para a captação do lixiviado de cada coluna, foram utilizados frascos com capacidade de $200 \mathrm{~mL}$, até que todo volume adicionado fosse captado a cada dia. De acordo com a quantidade total de água adicionada ao sistema, foram utilizados, para cada grupo, 59 frascos, sendo 29 frascos no primeiro dia e seis frascos a cada um dos cinco dias seguintes de coleta.

As concentrações de Mn nos extratos dos tratamentos e no lixiviado das colunas foram analisadas por "Plasma Acoplado Indutivo/ Espectroscopia de Emissão Óptica” (ICP-OES), utilizando um aparelho Perkin-Elmer, modelo Optima 3000, com limite de detecção (LD) de 0,010mg $\mathrm{kg}^{-1}$ e limite de quantificação (LQ) de 0,500 $\mathrm{mg} \mathrm{kg}^{-1}$ para Mn nos extratos da digestão de solo e com LD de 0,003mg L-1 e LQ de 0,010mg L-1, para Mn nos efluentes coletados nas colunas de solo. O limite de detecção (LD) do método foi calculado pela média dos valores dos brancos mais três vezes o desvio-padrão do branco de todas as análises (10 repetições). Para a validação da determinação do teor pseudototal de Mn no solo, utilizou-se um material certificado de referência (NIST SRM 2709, San Joaquin Soil), obtendo-se uma recuperação de 95\%, estando dentro dos limites estabelecidos pelo programa WEPAL-ISE e dos intervalos admitidos pelo NIST como normais para a amostra SRM 2709. O delineamento experimental usado foi inteiramente casualizado comparando o efeito da adição de escória de aciaria dentro de cada grupo de resíduo. As análises estatísticas foram feitas utilizando-se o teste de Tukey a 5\% para comparar médias na concentração de $\mathrm{Mn}$, no extrato solubilizado, em cada grupo de resíduos.

\section{RESULTADOS E DISCUSSÃO}

Concentração de Mn no solubilizado dos grupos (G1, G2 e G3) nos tratamentos

A maior dose do resíduo inertizante escória de aciaria reduziu a solubilidade do $\mathrm{Mn}$ a valores inferiores ao limite máximo permitido (ABNT, 2004c), nos grupos G1, G2 e G3 (Tabela 2).

$\mathrm{O}$ tratamento $\mathrm{G} 1+6 \%$ EA reduziu a concentração de Mn no solubilizado do G1 em 422 vezes e 1.848 vezes no G2. A escória de aciaria elevou o pH dos solos, causando a redução da solubilidade do Mn. Segundo TREVIZAM et al. (2010), o pH é um importante fator que controla a mobilidade de elementos traço no solo, influenciando diretamente a
Tabela 2 - Concentração de Mn no extrato solubilizado (NBR 10006) dos resíduos removidos do pátio de estocagem de minério da CSN (G1, G2 e G3), nos tratamentos com diferentes porcentagens de escória de aciaria.

\begin{tabular}{ll}
\hline Tratamento & $\mathrm{Mn}^{1}$ \\
\hline G1 (testemunha) & $\mathrm{mg} \mathrm{L}^{-1}$ \\
G1 + 4\%EA & $21,11 \mathrm{a}$ \\
G1 + 6\%EA & $0,94 \mathrm{~b}$ \\
G2 (testemunha) & $0,05 \mathrm{c}$ \\
G2 + 4\%EA & $18,48 \mathrm{a}$ \\
G2 + 6\%EA & $0,31 \mathrm{~b}$ \\
G3 (testemunha) & $0,01 \mathrm{c}$ \\
G3 + 4\%EA & 9,96 \\
G3 + 6\%EA & nd \\
\hline
\end{tabular}

${ }^{1}$ Médias seguidas de mesma letra não diferem significativamente pelo Teste de Tukey, $\mathrm{P}<0,05$, entre as diferentes doses de escória de aciaria dentro de cada grupo (G1, G2 e G3). nd= não detectado.

EA - Escória de Aciaria.

Limite ABNT, 2004b (0,1mg L $\left.{ }^{-1}\right)$

dinâmica de carbonatos e fosfatos do solo, o balanço de cargas dependentes de $\mathrm{pH}$ e, consequentemente, a solubilidade de elementos traço. A importância do pH na dinâmica de Mn em um substrato foi avaliada por SILBER et al. (2008), que observaram que a sua solubilidade foi predominantemente controlada por reações dependentes de $\mathrm{pH}$ e, em valores de $\mathrm{pH}$ abaixo de 6,0, a solubilidade de $\mathrm{Mn}$ aumentou significativamente. Para o G3, o tratamento com a menor dose de escória de aciaria (4\%) já foi suficiente para reduzir as concentrações de Mn no solubilizado para valores inferiores ao limite máximo permitido. O grupo G3 apresenta as menores concentrações de Mn no solubilizado e maior valor de $\mathrm{pH}$, quando comparado aos outros grupos (Tabela 1).

Ensaio de lixiviação em coluna

A concentração de $\mathrm{Mn}$ nos lixiviados na testemunha do grupo G1 variou de $291 \mathrm{mg} \mathrm{L}^{-1}$, no primeiro volume (V01), a $15 \mathrm{mg}^{-1}$ no último (V59) (Figura 1a). As elevadas concentrações de Mn lixiviadas nas colunas predizem elevados potenciais de contaminação de camadas subsuperficiais de solo e aquíferos.

As maiores concentrações de Mn no lixiviado ocorreram nos primeiros volumes lixiviados (Figura 1a), correspondendo a um volume de chuva de $250 \mathrm{~mm}$ dia $^{-1}$, tendendo a se estabilizar após os seguintes. Nos tratamentos G1+4\% EA; G1+6\% EA; $\mathrm{G} 1+6 \% \mathrm{EA}+\mathrm{BQ}$ as variações entre (V01 e V59) foram, respectivamente: $172 \mathrm{mg} \mathrm{L}^{-1}$ a $1,5 \mathrm{mg} \mathrm{L}^{-1}$; 


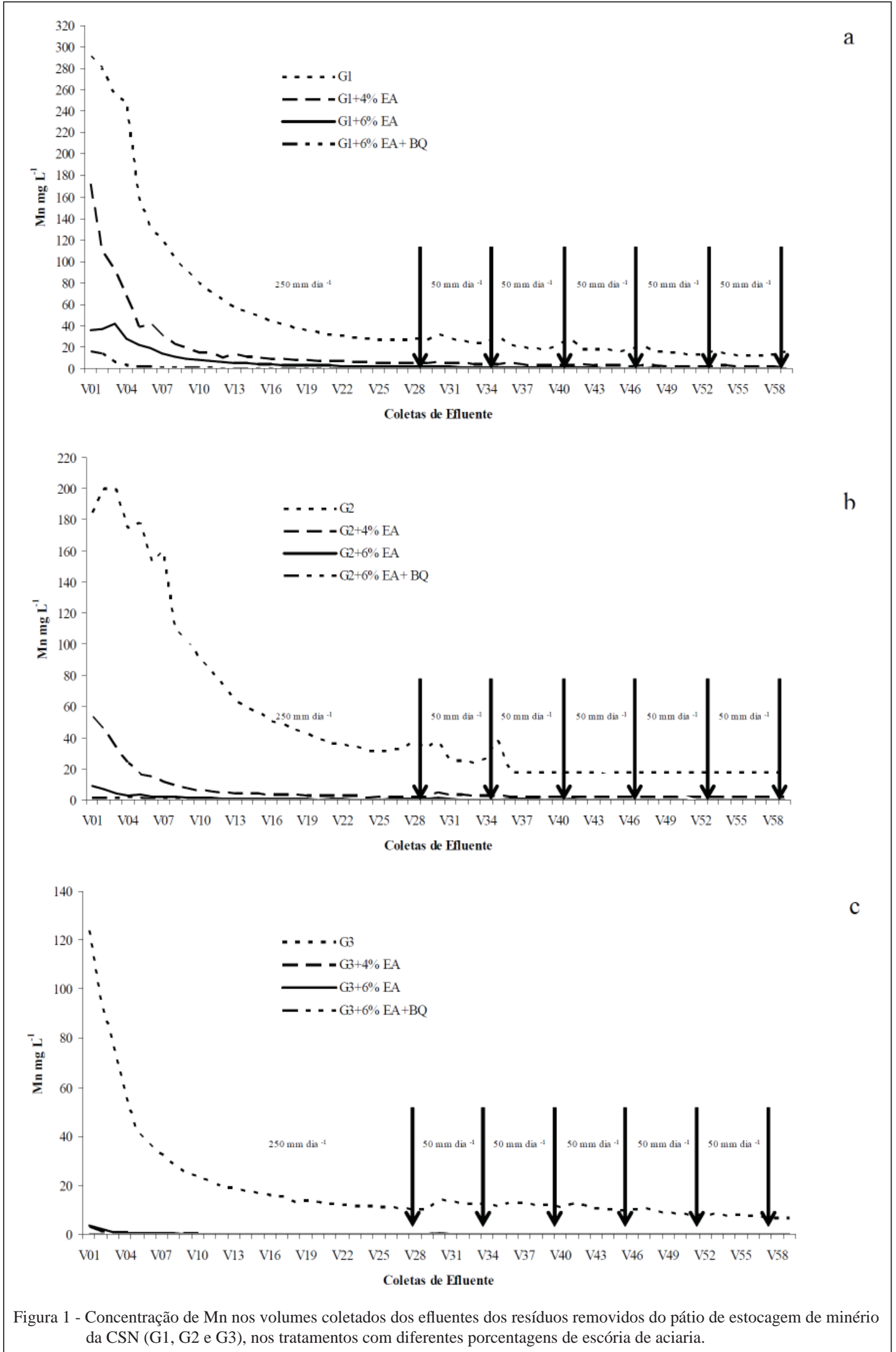

Ciência Rural, v.44, n.2, fev, 2014. 
36mg L $\mathrm{L}^{-1}$ a $0,2 \mathrm{mg} \mathrm{L}^{-1}$; $16 \mathrm{mg} \mathrm{L}^{-1}$ a $0,0 \mathrm{mg} \mathrm{L} \mathrm{L}^{-1}$. O tratamento $\mathrm{G} 1+4 \%$ EA reduziu a concentração de Mn do G1 em 41\% no (V01) e em 40\% no (V59), quando comparado à testemunha. O tratamento G1 + 6\% EA de 88\% no (V01) e em 100\% no (V59). Para o tratamento $\mathrm{G} 1+6 \% \mathrm{EA}+\mathrm{BQ}$, essa redução foi bastante pronunciada, atingindo $94,5 \%$ para o (V01) e 100\% para (V59). Neste tratamento, a partir do (V17) as concentrações de $\mathrm{Mn}$ nos efluentes foram iguais ou inferiores a $0,1 \mathrm{mg} \mathrm{L}^{-1}$. Entretanto, para o G1 sem tratamento, a concentração de Mn no (V59) ainda foi elevada, evidenciando a eficiência dos resíduos inertizantes na redução da mobilidade deste elemento.

Diversos estudos corroboram a importância do $\mathrm{pH}$ na redução da mobilidade de elementos traço no solo (NASCIMENTO et al., 2002; PUPATTO et al., 2004; AGOURAKIS et al., 2006; SANTOS et al., 2007). O G1 é o grupo que apresenta a maior acidez e maior concentração de Mn no solubilizado, com valor médio de 21,11mg L ${ }^{-1}$ (Tabela 1). A simulação realizada através de uma chuva acumulada de 500mm, a partir da saturação do solo, apresentou uma capacidade de solubilização superior ao do próprio teste de solubilização. Mesmo nessas condições muito críticas, o tratamento G1 + 6\% EA + BQ conseguiu reduzir a concentração de $\mathrm{Mn}$, a partir do (V17) (Figura 1a), a valores abaixo do máximo permitido (ABNT, 2004c). Essa redução na concentração de Mn no solubilizado ocorreu, possivelmente, pelo aumento das reações de precipitação do Mn na forma de carbonatos (ALLOWAY, 1995), além da adsorção específica na superfície dos cristais de argila e óxidos de ferro e alumínio, favorecidas pela elevação do $\mathrm{pH}$ de 3,6 para 7,0, nesse tratamento, e a presença de barreiras químicas (EA).

Os resultados encontrados para G2

(Figura 1b) foram semelhantes ao G1, entretanto, pode-se observar neste grupo, uma maior eficiência dos tratamentos quando comparados com G1 (Figura 1a), possivelmente devido aos menores teores pseudototais de $\mathrm{Mn}$ neste grupo e maiores porcentagens de argila (Tabela 1), partículas que estão diretamente relacionadas com as cargas elétricas do solo dependentes de $\mathrm{pH}$. A concentração de $\mathrm{Mn}$ nos efluentes do G2 sem tratamento variou de $184 \mathrm{mg} \mathrm{L}^{-1}$ no primeiro volume (V01) a $17 \mathrm{mg} \mathrm{L}^{-1}$ no último volume coletado (V59) (Figura 1b). Nos tratamentos $\mathrm{G} 2+4 \% \mathrm{EA}$; G2 + 6\%EA; e G2 + 6\%EA + BQ, as variações entre (V01 e V59) foram, respectivamente: 53mg L ${ }^{-1}$ a $1,8 m g \mathrm{~L}^{-1}$; $9 \mathrm{mg} \mathrm{L}^{-1}$ a $0,0 \mathrm{mg} \mathrm{L}^{-1}$; $1,68 \mathrm{mg} \mathrm{L}^{-1}$ a $0,0 \mathrm{mg} \mathrm{L}^{-1}$. O tratamento $\mathrm{G} 2+4 \%$ EA reduziu a concentração de Mn do G2 de $81 \%$ no (V01) e de 89,5\% no (V59), quando comparado à testemunha. $\mathrm{O}$ tratamento G2 + 6\% EA de 97\% no (V01) e de 100\% no (V59). Para o tratamento G2 + 6\% EA + BQ, essa redução foi muito elevada, atingindo 99,4\% no (V01) e 100\% no (V59). Neste tratamento, a partir do (V15), as concentrações de Mn nos efluentes foram iguais ou inferiores ao limite máximo permitido, que é de $0,1 \mathrm{mg} \mathrm{L}^{-1}$, segundo ABNT, 2004c. O tratamento G2+6\% EA atingiu valores iguais ou inferiores ao limite máximo permitido a partir do (V42), ratificando a importância do pH na imobilização de Mn no solo.

A concentração de Mn nos efluentes do G3 (Figura 1c) sem tratamento variou de $124,01 \mathrm{mg} \mathrm{L}^{-1}$ no primeiro volume (V01) a 6,83 $\mathrm{mg} \mathrm{L}^{-1}$ no último (V59). Nos tratamentos G3+4\% EA; G3+6\% EA; e G3+6\% $\mathrm{EA}+\mathrm{BQ}$, as variações entre (V01) e (V59) foram, respectivamente: $3,16 \mathrm{mg} \mathrm{L}^{-1}$ a $0,0 \mathrm{mg} \mathrm{L}^{-1} ; 3,74 \mathrm{mg} \mathrm{L}^{-1}$ a $0,0 \mathrm{mg} \mathrm{L}^{-1} ; 0,1 \mathrm{mg} \mathrm{L}^{-1}$ a $0,0 \mathrm{mg} \mathrm{L}^{-1}$. O tratamento G3+4\% EA reduziu a concentração de Mn do G3 de $97,5 \%$ no (V01) e de 100\% no (V59), comparado à testemunha. A concentração de Mn, neste tratamento, atingiu valores iguais ou inferiores ao limite máximo permitido a partir de (V33). O tratamento G3 + 6\% EA reduziu de $97 \%$ no (V01) e de 100\% no (V59). Para o tratamento G3 + 6\% EA + BQ, essa redução foi bastante pronunciada, atingindo $99,9 \%$ para o (V01) e 100\% para (V59). Neste tratamento, todos os efluentes coletados nos tratamentos usando escória de aciaria apresentaram concentrações de Mn iguais ou inferiores a $0,1 \mathrm{mg} \mathrm{L}^{-1}$. Essa maior eficiência na redução da lixiviação de Mn ocorreu, provavelmente, pelo fato de o G3 ser o grupo que apresentou maiores valores de $\mathrm{pH}(6,7)$ e as menores concentrações de Mn no solubilizado (Tabela 1).

\section{CONCLUSÃO}

A escória de aciaria foi eficiente na redução da solubilidade de Mn nos solos estudados, sendo que o tratamento com $6 \%$ escória de aciaria + barreiras químicas foi o mais eficiente na redução da mobilidade de Mn em todos os grupos de solos estudados.

\section{REFERÊNCIAS}

ABNT (ASSOCIAÇÃO BRASILEIRA DE NORMAS TÉCNICAS). NBR-10007: amostragem de resíduos sólidos. Rio de Janeiro, 2004a. 21p.

ABNT(ASSOCIAÇÃO BRASILEIRADE NORMAS TÉCNICAS). NBR 10006: procedimento para obtenção de extrato solubilizado de resíduos sólidos. Rio de Janeiro, 2004b. 3p.

ABNT (ASSOCIAÇÃO BRASILEIRA DE NORMAS TÉCNICAS). NBR-10004: resíduos sólidos - Classificação. Rio de Janeiro, 2004c. 71p. 
AGOURAKIS, D.C. et al. Comportamento de Zn e Mn de pilhas alcalinas em uma coluna de solo. Química Nova, v.29, p.960964, 2006. Disponível em: <http://dx.doi.org/10.1590/S010040422006000500014>. Acesso em: 20 jun. 2012. doi: 10.1590/ S0100-40422006000500014.

ALLOWAY, B.J. Soil process and the behavior of metals. In: ALLOWAY, B.J. Heavy metals in soils. Glasgow: Blackie Academic \& Professional, 1995. p.11-37.

BASTA, N.T.; MCGOWEN, S.L. Evaluation of chemical immobilization treatments for reducing heavy metal transport in a smelter-contaminated soil. Environmental Pollution, v.127, p.73-82, 2004. Disponível em: <http://dx.doi.org/10.1016/S02697491(03)00250-1>. Acesso em: 12 abr. 2012. doi: 10.1016/S02697491(03)00250-1.

CASTALDI, P. et al. Heavy metal immobilization by chemical amendments in a polluted soil and influence on white lupin growth. Chemosphere, v.60, p.365-371, 2005. Disponível em: <http:// dx.doi.org/10.1016/j.chemosphere.2004.11.098>. Acesso em: 03 dez. 2011. doi: 10.1016/j.chemosphere.2004.11.098.

EPA (UNITED STATES ENVIRONMENTAL PROTECTION AGENCY). Acid digestion of sediments, sludges, and soils Method 3050b. Washington: EPA, 1986. 12p.

FUNGARO, D.A. et al. Estabilização de solo contaminado com zinco usando zeólitas sintetizadas a partir de cinzas de carvão. Química Nova, v.27, p.582-585, 2004. Disponível em: <http:// dx.doi.org/10.1590/S0100-40422004000400012>. Acesso em: 26 jun. 2013. doi: 10.1590/S0100-40422004000400012.

MAGALHÃES, M.O.L. et al. Desempenho de duas espécies de eucalipto em solo com elevados teores de Mn. Revista Brasileira de Engenharia Agrícola e Ambiental, v.16, p.9298, 2012. Disponível em: <http://dx.doi.org/10.1590/S141543662012000100012>. Acesso em: 12 mar. 2013. doi: 10.1590/ S1415-43662012000100012.
MESQUITA, A.A. et al. Remediação de solos tratados com lodo rico em Zn. Revista Brasileira de Engenharia Agrícola e Ambiental, v.10, p.738-744, 2006. Disponível em: <http://dx.doi. org/10.1590/S1415-43662006000300029>. Acesso em: 11 nov. 2011. doi: 10.1590/S1415-43662006000300029.

NASCIMENTO, C.W.A. et al. Dessorção, extração e fracionamento de manganês em latossolos. Revista Brasileira de Ciência do Solo, v.26, p.589-597, 2002. Disponível em: <http://sbcs.solos.ufv. br/solos/revistas/v26n3a03.pdf>. Acesso em: 15 ago. 2012.

SANTOS, F.S. et al. Chemical amendment and phytostabilization of an industrial residue contaminated with $\mathrm{Zn}$ and $\mathrm{Cd}$. Scientia Agricola, v.64, p.506-512, 2007. Disponível em: <http://dx.doi. org/10.1590/S0103-90162007000500008>. Acesso em: 13 abr. 2012. doi: 10.1590/S0103-90162007000500008.

SILBER, A. et al. Kinetics and mechanisms of pH-dependent $\mathrm{Mn}(\mathrm{II})$ reactions in plant-growth medium. Soil Biology \& Biochemistry, v.40, p.2787-2795, 2008. Disponível em: <http:/ dx.doi.org/10.1016/j.soilbio.2008.07.026>. Acesso em: 27 jun. 2013. doi: 10.1016/j.soilbio.2008.07.026.

PRADO, R.M. et al. Efeito residual da escória de siderurgia como corretivo de acidez do solo na soqueira de cana-deaçucar. Revista Brasileira de Ciência do Solo, v.27, p.287296, 2003. Disponível em: <http://dx.doi.org/10.1590/S010006832003000200009>. Acesso em: 12 mar. 2013. doi: 10.1590/ S0100-06832003000200009.

PUPATTO, J.G.C. et al. Atributos químicos do solo, crescimento radicular e produtividade do arroz de acordo com a aplicação de escórias. Pesquisa Agropecuária Brasileira, v.39, p.12131218, 2004. Disponível em: <http://dx.doi.org/10.1590/S0100204X2004001200008>. Acesso em: 08 mar. 2013. doi: 10.1590/ S0100-204X2004001200008.

TREVIZAM, A.R. et al. Aplicação de fósforo para imobilização química do cádmio em solo contaminado. Química Nova, v.33, p.1235-1241, 2010. Disponível em: <http://dx.doi.org/10.1590/ S0100-40422010000600003>. Acesso em: 25 maio 2012. doi: 10.1590/S0100-40422010000600003. 